\title{
Pole Placement Design of Power Control Algorithms
}

\author{
Fredrik Gunnarsson, Fredrik Gustafsson and Jonas Blom \\ Department of Electrical Engineering \\ Linköpings universitet, SE-581 83 Linköping, Sweden \\ WWW: http://wWw. control.isy.liu.se \\ Email: fred@isy.liu.se, fredrik@isy.liu.se, \\ jb@isy.liu.se
}

April 16, 1999

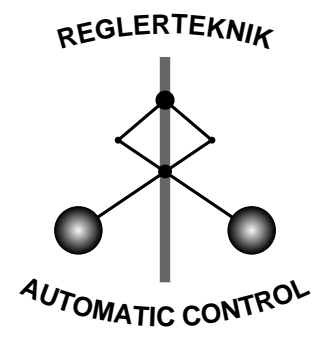

LINKÖPING

Report no.: LiTH-ISY-R-2149

Submitted to VTC'99, Houston, TX, USA

Technical reports from the Automatic Control group in Linkping are available by anonymous ftp at the address ftp.control.isy.liu.se. This report is contained in the compressed postscript file 2149.ps.Z. 



\begin{abstract}
Time delays reduces the performance of any controlled system. If neglected in the design phase, the system may even become unstable when using the designed controller. Several power control strategies have been proposed in order to improve the capacity of cellular radio systems, but time delays are usually neglected. Here, it is shown that the problems can be handled by considering the time delays in the design phase in order to choose the appropriate parameter values. Most popular algorithms can be seen as special cases of an integrating controller. This structure is extended first to a PI-controller and then further on to a general linear controller of higher orders. Corresponding design procedures are outlined based on techniques, such as pole placement, from the field of automatic control. The PI-controller is a very appealing choice of structure, with better performance compared to an I-controller and less complex than a higher order controller. The benefits are further illuminated by network simulations.
\end{abstract}

Keywords: Cellular radio systems; Power Control Algorithms; Pole placement; Local loop design; Linear controllers 


\section{Introduction}

For proper operation of a high-capacity cellular radio system, power control is an essential feature. Among others, there are three important aspects to consider.

- Extracting relevant information from the available measurements.

- Design a linear power control algorithm and tune the parameter for optimized performance.

- Incorporate nonlinear components to handle constraints and priorities.

The second issue is dealt with here, while the others are discussed in $[12,13]$. Several transmitter power control algorithms have been proposed to date. Most schemes strive to balance the carrier-to-interference ratios $(\mathrm{C} / \mathrm{I})$ on each channel such that every mobile or base station achieve the same $\mathrm{C} / \mathrm{I}[17]$.

To avoid extensive control signaling in the network, it is desirable to use distributed algorithms, where the transmitter powers are locally controlled based on local measurements (e.g. C/I). Such distributed algorithms have previously been studied in $[8,7,1,16,14,2,15]$.

These algorithms perform well in rather ideal cases, but in real systems there are a number of effects that hamper the performance as discussed in $[5,6,10]$. Among the most troublesome are the effects from time delays in the system. The stability analysis in $[5,11]$ reveals that the Distributed Power Control (DPC) algorithm $[8,7]$, which works fine under ideal circumstances, yields an unstable system when subject to a small time delay.

When the time delay is identified, the information can be considered not only in stability analysis, but also in the design phase in order to reduce its effects as much as possible. Most popular algorithms can be seen as special cases of an integrating controller [5], which is generalized into a Proportional Integrating (PI) controller. Then, the issue of performance measures and specifications are discussed. Techniques from the field of automatic control, such as pole placement design will be reviewed and applied. As always, the solution can be seen as a tradeoff between ability to track fast variations and to attenuate disturbances. Some illuminating simulations and conclusions conclude the paper.

\section{System Model}

Signal gains and power levels can be expressed using either logarithmic (e.g. $d B$ or $d B m)$ or linear scale. To avoid confusion we will employ the convention of indicating linearly scaled values with a bar. Thus $\bar{g}_{i j}$ is a value in linear scale and $g_{i j}$ the corresponding value in logarithmic scale.

Assume that the $m$ mobile stations on a specific radio channel are transmitting using the powers $p_{i}(t), i=1, \ldots, m$. The signal between mobile station 
$i$ and base station $j$ is attenuated by the signal gain $g_{i j}(t)(<0)$. Thus the corresponding connected base station will experience a desired carrier signal $C_{i}(t)=p_{i}(t)+g_{i i}(t)$ and an interference plus noise $I_{i}(t)$ (values in logarithmic scale). The carrier-to-interference ratio at base station $i$ is defined by

$$
\gamma_{i}(t)=p_{i}(t)+g_{i i}(t)-I_{i}(t) .
$$

We will assume that the Quality of Service (QoS) is depending only on the C/I, and is acceptable iff

$$
\gamma_{i}(t) \geq \gamma^{*}, i=1, \ldots, m .
$$

The measured (or estimated) C/I:s, denoted by $\hat{\gamma}_{i}$, may be inaccurate or corrupted by noise. Therefore various filters $F(q)$ can be used to obtain smooth estimates. As concluded in [11], an exponential forgetting (EF) filter is an interesting choice. It is defined by the following recursion

$$
\gamma_{i}(t+1)=\lambda \gamma_{i}(t)+(1-\lambda) \hat{\gamma}_{i}(t+1), \quad 0 \leq \lambda<1,
$$

or using the delay operator $q\left(q^{-n} \gamma(t)=\gamma(t-n)\right)$

$$
\gamma_{i}(t)=\frac{(1-\lambda) q}{q-\lambda} \hat{\gamma}_{i}(t)=F_{E F}(q) \hat{\gamma}_{i}(t) .
$$

Primarily, there are two motives for considering the filter. On the one hand, it can be introduced as a device to reduce the noise. The parameter $\lambda$ is then a design variable. On the other hand, it can be seen as a simple model of an estimator, when studying its effects on the dynamics of the power control loop. Then $\lambda$ is a fixed value, which should be identified by experiments or modeling.

The output powers are considered to be unconstrained. Appropriate modifications when considering constrained output powers are discussed in $[2,9,13]$.

A general distributed power control algorithm $R(q)$ and its surroundings can be depicted as in Figure 1, and this loop will be referred to as the local loop. Since the focus is on a single connection, the index $i$ will be dropped for clarity. The power outputs and measurements are delayed by $n_{p}$ and $n_{m}$ samples respectively. In this work, the typical situation $n_{p}=n_{m}=1$ will be studied. The measurement noise or estimation error $v(t)$ is a nuisance signal . Moreover, the objective is to track a target signal (possibly constant) $\gamma_{t g t}(t)$. From (2) it is natural to choose $\gamma_{t g t}(t) \geq \gamma^{*}$.

\section{Controller Structures}

\subsection{I-controller}

Many of the algorithms discussed in previous work, e.g. the DPC algorithm [8, 7], the Constant Received Power (CRP) algorithm [2] and the algorithms proposed in $[16,14,15]$, can be shown [5] to be special cases of the integrating 


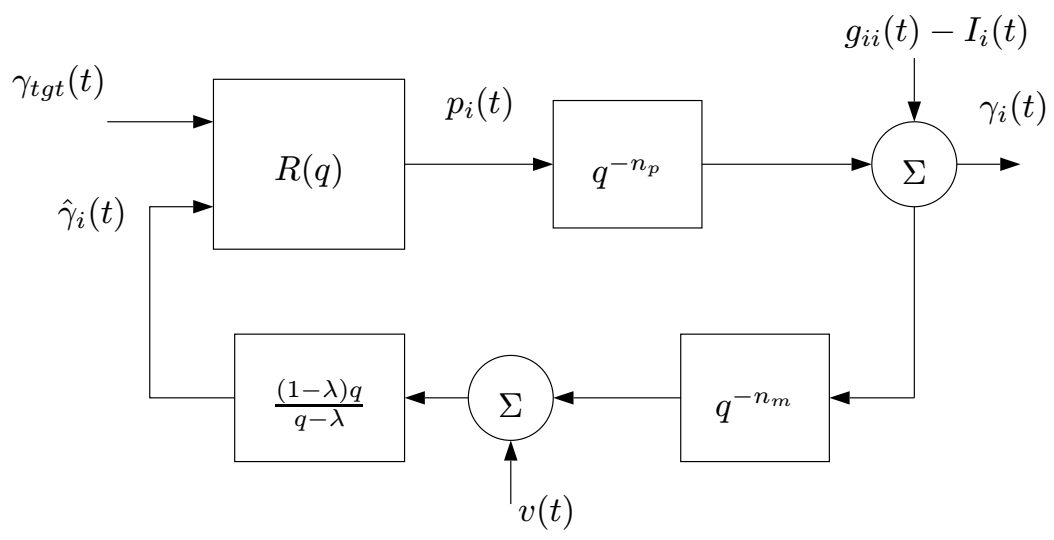

Figure 1: The local loop when employing the general power control algorithm $R(q)$ and the smoothing filter $F_{E F}(q)=\frac{(1-\lambda) q}{q-\lambda}$.

controller

$$
p(t+1)=p(t)+K_{i} T_{s}\left(\gamma_{t g t}(t)-\hat{\gamma}(t)\right),
$$

where $T_{s}$ is the sampling interval and $K_{i}$ is a design parameter. Define the error $e(t)=\gamma_{t g t}(t)-\hat{\gamma}(t)$ and using the delay operator, the I-controller can be expressed as

$$
p(t)=\frac{K_{i} T_{s} q}{q-1} e(t)=R_{I}(q) e(t)
$$

where an extra "q" is added to make the controller delay-less, and let $n_{m}$ and $n_{p}$ capture the delays.

\section{$3.2 \quad$ PID-controller}

A natural extension of (4) is the Proportional Integrating Differentiating (PID) [3] controller often considered in process applications. It can be described by

$$
\begin{aligned}
e(t) & =\left(\gamma_{t g t}(t)-\hat{\gamma}(t)\right) \\
x(t+1) & =x(t)+K_{i} T_{s} e(t) \\
p(t) & =K_{p} e(t)+x(t+1)+\frac{K_{d}}{T_{s}}(e(t)-e(t-1)),
\end{aligned}
$$

where $x(t)$ is the integration state, and $K_{p}, K_{i}$ and $K_{d}$ are parameters. It is common to omit the differentiation $\left(K_{d}=0\right)$ when the measurements are noisy. An alternative is to apply approximated differentiation [3]. In this paper, differentiation will be omitted. Using the delay operator, we obtain

$$
p(t)=\frac{\left(K_{p}+K_{i} T_{s}\right) q-K_{p}}{q-1} e(t)=R_{P I}(q) e(t) .
$$




\subsection{General Linear Controller}

A more general linear controller is given by

$$
p(t)=\frac{B_{f f}(q)}{A_{f f}(q)} \gamma_{t g t}(t)-\frac{B_{r}(q)}{A_{r}(q)} \hat{\gamma}(t),
$$

where $A_{f f}, B_{f f}, A_{r}$ and $B_{r}$ are polynomials in the delay operator. By introducing the following notation, this general control loop can be depicted as in Figure 2

$$
\frac{B(q)}{A(q)}=\frac{1}{q}, \frac{B_{F}(q)}{A_{F}(q)}=\frac{(1-\lambda)}{q-\lambda}
$$

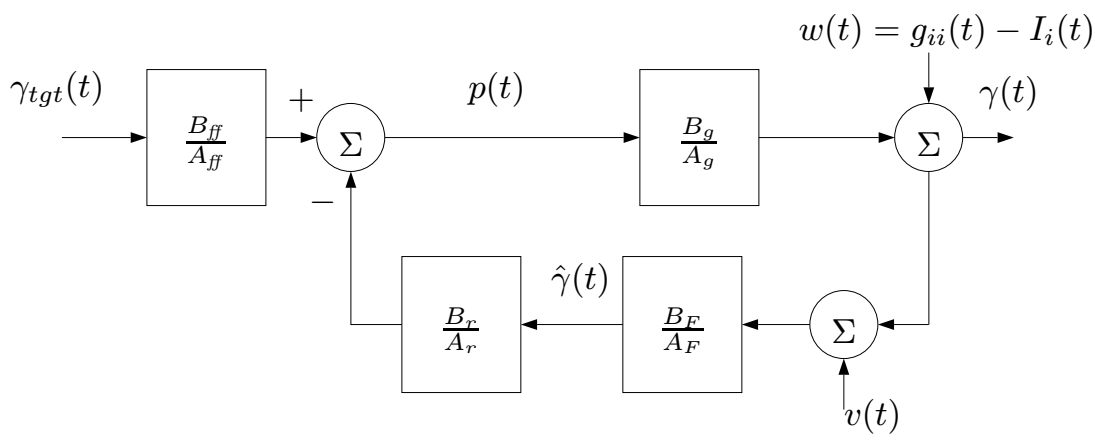

Figure 2: The general power control problem in local loop.

\section{Properties of Linear Systems}

The presentation here will be rather compact. For further details, see [4]. Consider the continuous-time system

$$
y(t)=G_{c}(p) u(t)=\frac{B_{c}(p)}{A_{c}(p)} u(t),
$$

where $p$ is the differentiation operator $p u(t)=\dot{u}(t)$. The response to changes in $u(t)$ are described by the locations of the poles $s_{j}$ of $G_{c}(p)$, i.e. the roots of the polynomial $A_{c}(p)$. As a rule of thumb, the response is acceptable if the poles are located within the shaded area of Figure 3a. The speed of reaction is determined by the distance to the origin, and the slightly oscillatory behavior during settling, by the angle to the negative real axis. The poles closest to the origin will dominate the behavior and are therefore referred to as dominating poles.

When discretizing the continuous system,the corresponding discrete-time system $G_{d}(q)$ will have poles at

$$
z_{j}=e^{s_{j} T_{s}} .
$$


In addition, the poles will result in a number of zeros, i.e. roots to the numerator polynomial. However, a good approximation for systems of low order is that the characteristics are determined by the poles to $G_{d}(q)$ and specifications can be stated in the continuous-time domain using (8). Mapping the area in Figure 3a using (8) yields the area in Figure 3b.

Consider the system in Figure 2. The closed-loop system is obtained from straightforward computations as (where the argument $q$ has been suppressed)

$$
\gamma(t)=\frac{A_{g} A_{r} A_{F}}{A_{g} A_{r} A_{F}+B_{g} B_{r} B_{F}}\left(\frac{B_{g} B_{f f}}{A_{g} A_{f f}} \gamma_{t g t}(t)+w(t)+\frac{B_{g} B_{r}}{A_{g} A_{r}} v(t)\right)
$$

From this expression, we see that the design is all about choosing the appropriate polynomials in order to place the poles and zeros at desired locations. Note that the characteristic polynomial $P=A A_{r} A_{F}+B B_{r} B_{F}$ plays an important role. When simplifying expressions like in (9) it is important to remember only to cancel factors that are well-damped, i.e. have roots within the shaded area in Figures 3ab.

A fundamental result from control theory, is that we need an integration in the open-loop system in order to drive the error to zero in steady-state. Therefore it may be desirable to introduce integration in the controller, i.e. $(q-1)$ is a factor of $A_{r}$.

\section{Design}

The design will be treated separately for each of the structures in Section 3

b.

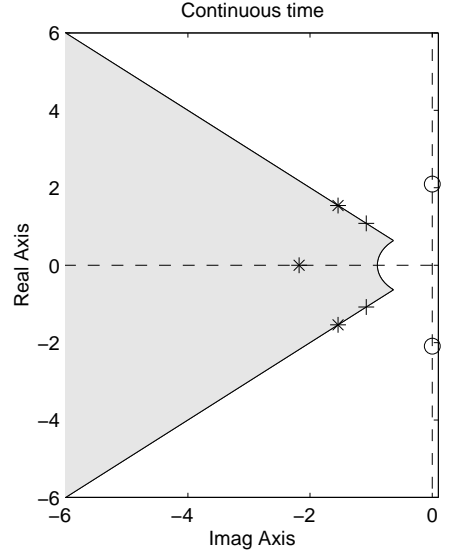

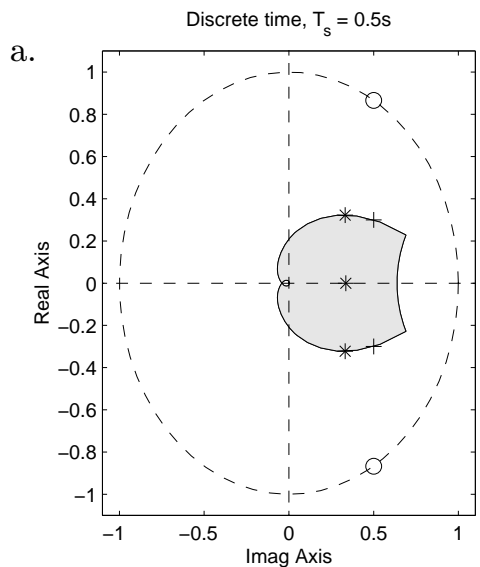

Figure 3: Poles located in the shaded area corresponds to acceptable behavior of the system in continuous time (a) and discrete time (b). The 'o':s correspond to the use of the DPC algorithm, the '+':s to an optimized I-controller, and the '*':s to an optimized PI-controller. In this case no smoothing filter is considered $(\lambda=0)$. 


\subsection{I-controller}

In this case, the characteristic polynomial is given by

$$
P(q)=q^{2}-(1+\lambda) q+K_{i} T_{s}(1-\lambda),
$$

which is easy to solve in terms of $\lambda$. Assume that we prioritize fast responses, the corresponding dominating continuous-time poles should be as distant as possible from the origin and within the shaded area. The resulting pole locations when $\lambda=0$ are found in Figure 3. For comparison, the poles when employing the DPC algorithm are plotted.

\section{$5.2 \quad$ PI-controller}

Since we now have two degrees of freedom, we expect to be able to do more. The characteristic polynomial is given by

$$
\begin{aligned}
P(q)= & q^{3}-(1+\lambda) q^{2}+\left(\left(K_{p}+K_{i} T_{s}\right)(1-\lambda)-\lambda\right) q- \\
& K_{p}(1-\lambda) .
\end{aligned}
$$

For fastest possible response, we require to place the corresponding continuoustime poles at

$$
s=r_{0}\left[e^{i \pi(1-\delta)}, e^{i \pi}, e^{i \pi(1+\delta)}\right],
$$

where $r_{0}$ is the distance to the origin and $0 \leq \delta \leq 0.25$ is a design parameter describing the angle to the negative real axis. Using Equations (8) and (11), the corresponding desired characteristic polynomial in discrete time is obtained. After some simplifications and a comparison with (10), we see that the optimal distance to the origin is obtained by (numerically) solving the following equation for $r_{0}$

$$
e^{-r_{0} T_{s}}+e^{-r_{0} T_{s} \cos (\pi \delta)} \cos \left(r_{0} T_{s} \sin (\pi \delta)\right)-1-\lambda=0
$$

The controller parameters are then computed as

$$
\begin{aligned}
K_{p}= & \frac{1}{1-\lambda}\left(e^{-r_{0} T_{s}(1+2 \cos (\pi \delta))}\right) \\
K_{i}= & \frac{1}{T_{s}(1-\lambda)}\left[\lambda+e^{-2 r_{0} T_{s} \cos (\pi \delta)}+\right. \\
& \left.e^{r_{0} T_{s}(1+\cos (\pi \delta))} \cos \left(r_{0} T_{s} \sin (\pi \delta)\right)\right]-\frac{K_{p}}{T_{s}}
\end{aligned}
$$

The resulting pole locations are found in Figure 3 for the case $\lambda=0$.

\subsection{General Linear Controller}

The polynomials $A_{f f}$ and $B_{f f}$ will be discussed further in the end and are treated as equal to unity initially. In the general case, we are able to place the close-loop 
poles at arbitrary locations. However, when the order of the system is higher, the zeros of the system, will affect the behavior. Thus, we have to place the zeros as well.

Consider the characteristic polynomial from (9)

$$
\begin{aligned}
P(q)= & (1-\lambda) B_{r}(q)+q(q-\lambda) A_{r}(q)= \\
& B(q) B_{r}(q)+A(q) A_{r}(q) .
\end{aligned}
$$

The design problem breaks down to find $A_{r}$ and $B_{r}$ so that the left side of (12) is equal to the specified $P(q)$. This Diophantine equation [4] has a solution if

$$
\begin{aligned}
\operatorname{deg}\{B\} & \leq \operatorname{deg}\{A\} \\
\operatorname{deg}\left\{A_{r}\right\} & \geq \max (\operatorname{deg}\{A\}-1, \operatorname{deg}\{B\}) \\
\operatorname{deg}\left\{B_{r}\right\} & =\operatorname{deg}\{A\}-1
\end{aligned}
$$

Note that if $A_{r}^{0}$ and $B_{r}^{0}$ is a solution, so is

$$
A_{r}=A_{r}^{0}+Q B, B_{r}=B_{r}^{0}-Q A,
$$

where $Q(q)$ is a general polynomial. Integration is easily employed by introducing

$$
A_{r}=(q-1) \tilde{A}_{r}, \tilde{A}=(q-1) A,
$$

and solve $P(q)=B(q) B_{r}(q)+\tilde{A}(q) \tilde{A}_{r}(q)$ instead of (12).

In the design, start by designing $A_{r}$ and $B_{r}$ for acceptable noise attenuation of $v(t)$ and the fast variations in $w(t)$ that we cannot track. Then use the polynomials $A_{f f}$ and $B_{f f}$ to shape the transfer function for tracking $\gamma_{t g t}(t)$. Remember to only cancel factors corresponding to well damped roots (i.e. in the shaded area in Figure 3.

\subsection{Discussion}

It may seem appealing to be able to place the poles arbitrarily, but it is important to note that the many degrees of freedom also is a curse in the design procedure, since it also involves placing zeros. When complexity is an issue, the simplicity of the PI-controller is appealing and it is often sufficient. However, when statistics of the disturbances are well known, this can more extensively be used in the design of a controller of higher order.

\section{Simulations}

The performance of different controller structures are illustrated using simple global network simulations. The scenario reflects the reactions of four mobile stations with settled powers, when a fifth mobile station establishes a call, initially using maximum power. No noise $v(t)$ is applied, the gains $g_{i j}$ are constant, and the mobiles remain fixed throughout the simulations. The I- and 
PI-controllers in Figure 3b both have poles in the shaded area, but as indicated by the simulations in Figure 4, the PI-controller corresponds to a more appealing response.

a.
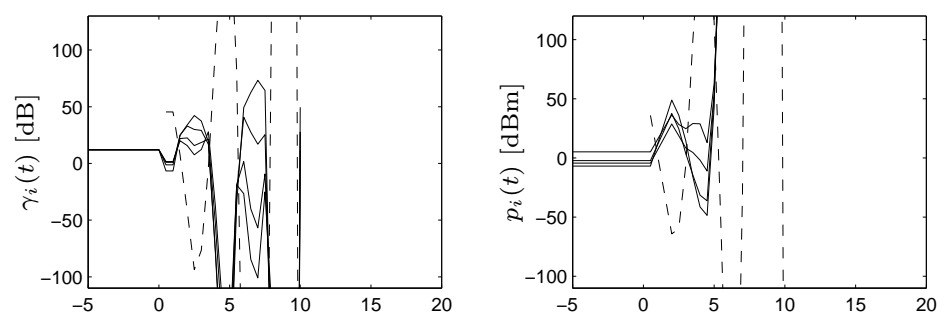

b.
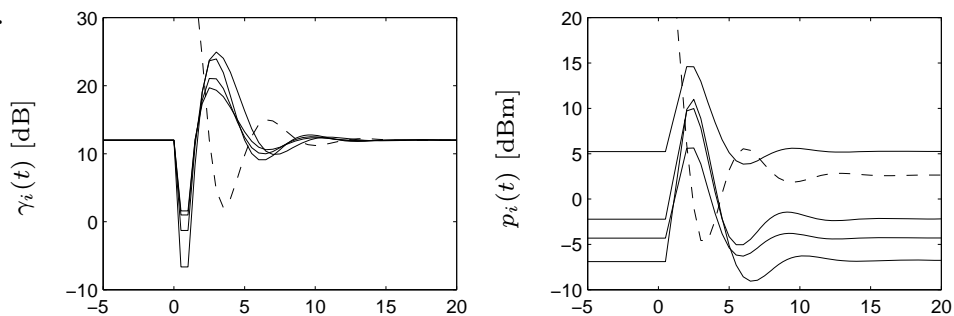

c.
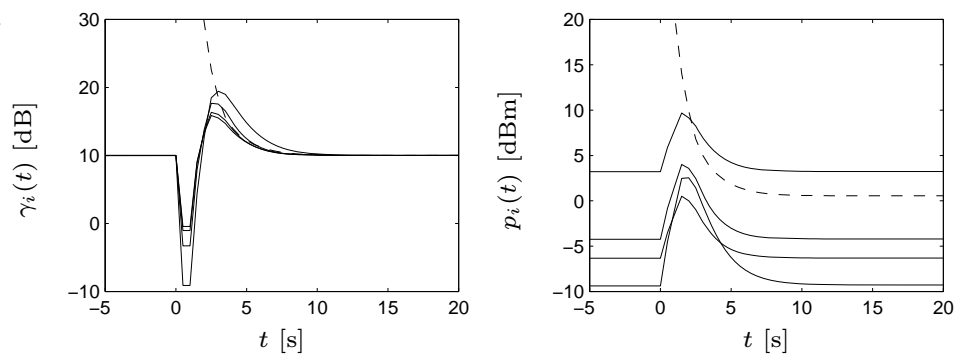

Figure 4: CIR (left) and output powers (right) from simulations. a) DPC algorithm, b) I-controller, c) PI-controller.

Furthermore, the different algorithms were employed in a more comprehensive simulator (see [12] for some and [5] for more details). The signal gains were affected by fading (slow and fast), and the 27 channels were distributed in a $3 / 9$ reuse pattern. Pseudo-random frequency hopping was applied, reducing the effects of fast fading. A sample interval of $0.48 \mathrm{~s}$ was used; same as the power control interval in GSM. The quality was measured using C/I, and a user is considered satisfied when having a mean $\mathrm{C} / \mathrm{I}$ above $\gamma^{*}=10 \mathrm{~dB}$. As seen in Figure 5, the PI-controller is again the most appealing choice.

\section{Conclusions}

Time delays reduces the performance of any controlled system. If neglected in the design phase, the system may even be unstable using the designed controller. 


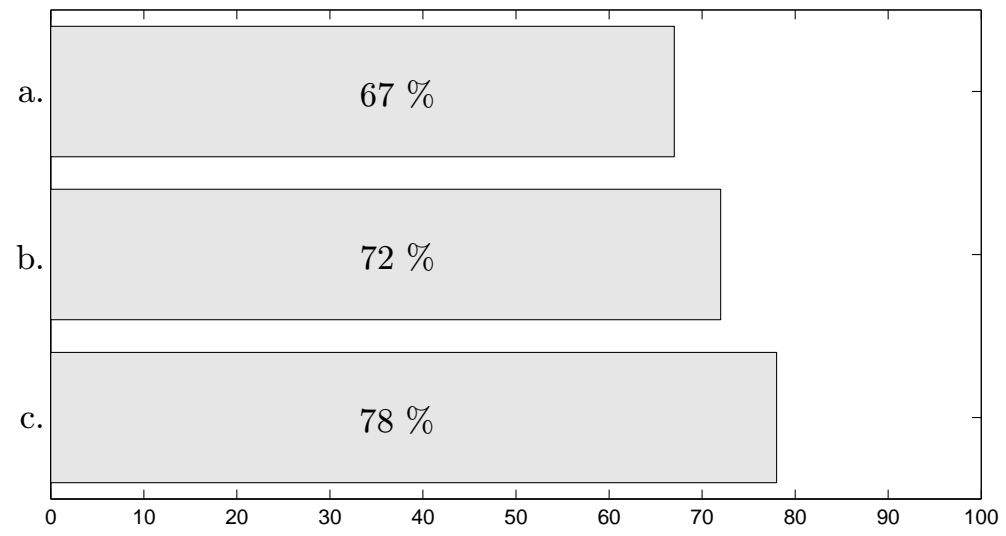

Figure 5: The percentage of satisfied users. a) An I-controller, b) an optimized I-controller, c) an optimized PI-controller.

In this work, techniques from the field of automatic control, such as pole placement, are reviewed. Time delays when identified are naturally considered in the design phase. The pole placement is more intuitive in continuous-time, and using a pole transformation from continuous to discrete-time poles, this intuition is utilized for performance specifications.

Three basic controller structures have been discussed in operation with an optional smoothing filter. Most discussed algorithms can be seen as special cases of an integrating controller and this is therefore a first choice. The natural extension to a PI-controller is discussed, as well as a general linear controller of higher order. In most cases, the PI-controller represents a relevant tradeoff between performance and complexity. However, when the statistics of the disturbances is well known, a more general structure is required. 


\section{References}

[1] M. Almgren, H. Andersson, and K. Wallstedt. Power control in a cellular system. In Proc. IEEE Vehicular Technology Conference, Stockholm, Sweden, June 1994.

[2] E. Anderlind. Resource Allocation in Multi-Service Wireless Access Networks. PhD thesis, Radio Comm. Systems Lab., Royal Inst. Technology, Stockholm, Sweden, October 1997.

[3] K. Åström and T. Hägglund. PID Controllers: Theory, Design, and Tuning. Instrument Society of America, Research Triangle Park, NC, USA, second edition, 1995.

[4] K. Åström and B. Wittenmark. Computed Controlled Systems - Theory and Design. Prentice-Hall, Englewood Cliffs, NJ, USA, 1984.

[5] J. Blom and F. Gunnarsson. Power Control in Cellular Radio Systems. Licenciate Thesis, Linköpings universitet, Sweden, June 1998. Available at http://www. control.isy.liu.se.

[6] J. Blom, F. Gunnarsson, and F. Gustafsson. A new concept of power control in cellular systems reflecting challenges of today's systems. In Proc. IEEE Global Telecommunications Conference, Sydney, Australia, November 1998.

[7] G. J. Foschini and Z. Miljanic. A simple distributed autonomus power control algorithm and its covergence. IEEE Transactions on Vehicular Technology, 42(4), 1993.

[8] S. A. Grandhi, R. Vijayan, and D. J. Goodman. Distributed power control in cellular radio systems. IEEE Transactions on Communications, 42(2), 1994.

[9] S. A. Grandhi, J. Zander, and R. Yates. Constrained power control. Wireless Personal Communications, 2(1), 1995.

[10] F. Gunnarsson, J. Blom, and F. Gustafsson. Method and system for quality-based power control in cellular communications systems. US Patent Application No. 09/031,635, 1998.

[11] F. Gunnarsson, J. Blom, and F. Gustafsson. Power control in cellular systems subject to constraints and time delays. In Proc. IEEE Global Telecommunications Conference, Sydney, Australia, November 1998.

[12] F. Gunnarsson, J. Blom, and F. Gustafsson. Estimation of the carrierto-interference ratio in cellular radio systems. In Proc. IEEE Vehicular Technology Conference, Houston, TX, USA, May 1999.

[13] F. Gunnarsson, F. Gustafsson, and J. Blom. Improved performance using nonlinear components in power control algorithms. In Proc. IEEE Vehicular Technology Conference, Houston, TX, USA, May 1999.

[14] T. H. Lee and J. C. Lin. A study on the distributed power control for cellular mobile systems. In Proc. IEEE Vehicular Technology Conference, Atlanta, GA, USA, April 1996. 
[15] L. Song and J. M. Holtzman. CDMA dynamic downlink power control. In Proc. IEEE Vehicular Technology Conference, Ottawa, Canada, May 1998.

[16] R. D. Yates. A framework for uplink power control in cellular radio systems. IEEE Journal on Selected Areas in Communications, 13(7), September 1995 .

[17] J. Zander. Performance of optimum transmitter power control in cellular radio systems. IEEE Transactions on Vehicular Technology, 41(1), February 1992. 